\title{
RATCHETS IN HOMOGENEOUS EXTENDED SYSTEMS: INTERNAL MODES AND THE ROLE OF NOISE
}

\author{
Angel Sánchez $^{*, \star}$, Luis Morales-Molina ${ }^{\dagger}$, Franz G. Mertens*, Niurka R. Quintero ${ }^{\ddagger}, \boldsymbol{\Upsilon}^{*}$ \\ Javier Buceta ${ }^{\S}$ and Katja Lindenberg $\$$ \\ * Grupo Interdisciplinar de Sistemas Complejos (GISC) and Departamento de Matemáticas, \\ Universidad Carlos III de Madrid, 28911 Leganés, Madrid, Spain \\ * Instituto de Biocomputación y Física de Sistemas Complejos (BIFI), Universidad de Zaragoza, \\ 50009 Zaragoza, Spain \\ ${ }^{\dagger}$ Physikalisches Institut, Universität Bayreuth, D-85440 Bayreuth, Germany \\ ${ }^{\ddagger}$ Departamento de Física Aplicada I, E. U. P., Universidad de Sevilla, Virgen de África \%, \\ 41011 Sevilla, Spain \\ ฯ Instituto Carlos I de Física Teórica y Computacional, Universidad de Granada, \\ 18071 Granada, Spain \\ $\S$ Department of Chemistry and Biochemistry, and Institute for Nonlinear Science, \\ University of California, San Diego, 9500 Gilman Drive, La Jolla, California 92093-0340, \\ U.S.A.
}

Received (received date)
Revised (revised date)
Accepted (accepted date)

We revisit the issue of directed motion induced by zero average forces in extended systems driven by ac forces. It has been shown recently that a directed energy current appears if the ac external force, $f(t)$, breaks the symmetry $f(t)=-f(t+T / 2), T$ being the period, if topological solitons (kinks) existed in the system. In this work, a collective coordinate approach allows us to identify the mechanism through which the width oscillation drives the kink and its relation with the mathematical symmetry conditions. Furthermore, our theory predicts, and numerical simulations confirm, that the direction of motion depends on the initial phase of the driving, while the system behaves in a ratchet-like fashion if averaging over initial conditions. Finally, the presence of noise overimposed to the ac driving does not destroy the directed motion; on the contrary, it gives rise to an activation process that increases the velocity of the motion. We conjecture that this could be a signature of resonant phenomena at larger noises.

Keywords: Ratchets, Rectification, Extended Systems, Solitons, Internal Modes, Noise, Activated Processes

\section{INTRODUCTION}

Within the exciting realm of transport phenomena in nonlinear systems [1], net directed motion induced by zero average forces is a very intriguing phenomenon that is being the subject of much research in the last ten years (see Ref. [2] for a 
review; see also Ref. [3] for an introductory presentation). The interest on this issue stems from the attempts to understand the way transport takes place inside cells through the so-called molecular motors [4]. Also, nanotechnology has motivated a number of works on this kind of phenomena, in view of its possible applications as current rectifiers [5]. Although the first works on this topic can be dated back to the beginning of the 20th century (see Sec. 1.2 in Ref. [2] for an interesting historical recollection), it was Feynman [6] who made the seminal contribution, after which the name ratchet is generally used to refer to this very general problem.

At a first stage, the ratchet phenomenon was studied in a zero-dimensional context, following the schemes proposed in Refs. $[7,8]$, in which a point particle was subjected to the action of a periodic, asymmetric potential and a periodic force [7] or, alternatively, the potential was periodically switched on and off [8]. Subsequently, the statistical physics community devoted a great deal of effort to the subject, and very many types of ratchet were proposed and studied (see Refs. [9] for a very short list of examples; see 2 for a very exhaustive bibliography).

The next step in the search for directed motion scenarios was to consider extended systems, focusing specially in soliton-bearing models (or, more generally, systems where nonlinear coherent excitations play a key role for the transport properties). Indeed, the fact that in many situations solitons behave very much like particles $[1,10]$ led immediately to search for generalizations of the one-degree-offreedom results to more (even infinite) degrees of freedom. Among the first works in this direction, we could mention Refs. [11,12,14], which studied solitons in a noisy environment. Again, much as in the case of point-like ratchets, these proposals stimulated both the statistical mechanics and the nonlinear science communities to look for further examples, not only theoretical [13], but also experimental and applied [15].

\section{Homogeneous extended ratchets}

Generally speaking, the appearance of ratchet-like behavior requires two ingredients: departure from thermal equilibrium (either by using correlated stochastic forces or deterministic forces) and breaking of spatial inversion symmetry [2], as mentioned above. This is actually the setup in the systems studied in the references cited so far and in a majority of other ratchet models. However, it has recently been realized that the use of an asymmetric driving can play the same role as the spatial asymmetry. Such an effect was first proposed for one-particle systems in Ref. [16] (see also Ref. 17). The analysis presented by Flach and coworkers indicated that a directed energy current appeared if $f(t)$ broke the symmetry $f(t)=-f(t+T / 2)$, $T$ being the period of the external driving. It was only natural then to try to verify this phenomenon in extended systems, and so it was done both in the quantum [18] and in the classical cases $[19,20]$. In this work, we revisit the example studied by these last authors, namely the ac driven, damped, sine-Gordon equation, given by

$$
\phi_{t t}-\phi_{x x}+\sin (\phi)=-\beta \phi_{t}+f(t) .
$$

In Refs. $[19,20]$, the previous symmetry considerations were generalized to the extended model (12). Again, it was found that if $f(t)$ broke the symmetry $f(t)=$ $-f(t+T / 2)$, and the total topological charge in the system was nonzero, a directed 
current should be observed whose direction and magnitude depended on the driving and damping parameters. It is important to realize that the condition on the topological charge implies that at least one kink or one antikink must exist, and that the numbers of both types of nonlinear excitations should differ at least by 1 . In the case when there is just one kink (or antikink) in the system, we are faced with an analogy with the point-like ratchet proposed in Ref. [16]. Indeed, as in many other instances [10], kinks behave basically as point-like particles, and the fact that their presence in the extended system is needed to have directed current reinforces this analogy. However, as we will see immediately, the scenario is not that simple, and in fact the point-like particle picture is not enough to understand the phenomenology.

As their working example, Flach et al. [19] and Salerno and Zolotaryuk [20] considered $f(t) \equiv \epsilon_{1} \cos (\delta t)+\epsilon_{2} \cos (m \delta t+\theta)$. For this choice, they performed numerical simulations that confirmed the symmetry analysis results. In view that the system did exhibit ratchet-like behavior, i.e., it rectified ac current, as kinks moved towards one direction in space, they tried a collective coordinate approach (see Ref. [10] for a review on this technique), in which the kink motion was reduced to a description in terms of an ordinary differential equation for the motion of its center. However, their attempt turned out to be unsuccesful [19,20], showing that, as advanced above, there was more to the net directed motion observed than the analogy to the point-like ratchet.

\section{What's wrong with this particle?}

At this point, it is interesting to discuss in some detail the context in which this research should be understood from the viewpoint of kink dynamics. A few years earlier, some of us [21] have proven that sine-Gordon kinks can not move when subjected to single-harmonic, ac forces plus damping. This had been debated during the nineties but the issue was finally settle down in the negative sense. For that problem, a collective coordinate description in terms of the motion of the kink center showed a quantitative agreement with the numerical simulation results. In the absence of damping, net directed motion was possible with single-harmonic forces, and again the phenomenon was very accurately captured by the collective coordinate approach.

In a subsequent work, we applied the same approach to a related system, the so-called $\phi^{4}$ equation $[22,23]$. In this case, we found that, in spite of the fact that for most parameter choices the kink behavior was correctly described by a particle picture, there were anomalous resonances that could only be described by taking into account that $\phi^{4}$ kinks possess an internal mode, namely an oscillation of the kink shape arising from simple linear stability analysis. To include such an internal mode in the theory, we resorted to an improved collective coordinate approach with two variables, namely the position and the width of the kink, thus recovering the complete agreement with the simulations.

In view of this, it seems natural to think that something similar was taking place when the sine-Gordon system was forced with two harmonics. In the original works $[19,20]$, the authors proposed a similar explanation for the failure of their approach. An early attempt to develop a two-variable theory for this problem [24] failed as 
well, as it could only provide a partial answer indicating that it was indeed necessary that the kink width was a dynamical variable. Furthermore, the correct description of the dynamics and the connection to the symmetry analysis were still lacking. But there was yet another problem in this line of reasoning: that sine-Gordon kinks do not exhibit internal modes, as was shown in Ref. [25]. Nevertheless, such a conundrum might be solved if we realized that, in fact, the sine-Gordon equation we are studying is forced, and it has been shown [26] that external forces can excite phonons (linear radiation waves) that give rise to a total effect similar to that of an internal mode. Thus, it was later shown in the case of the soliton ratchet proposed by Salerno and Quintero (an inhomogeneous sine-Gordon equation, see Ref. [14]) that the crucial factor was the interaction of that effective internal mode and the translation mode. Another instance where the possible role of internal modes had been previously highlighted was Ref. [27], where a two-particle system with an internal degree of freedom was studied, exhibiting ratchet behavior in symmetric potentials. In view of all this, the possibility of trying to incorporate an internal mode in the description of this problem seemed to be an intriguing issue. This we discuss in the next section. Some of the results below were presented in a much more concise form in Ref. [28].

\section{Including the internal mode}

In the following, we consider again the system proposed in Refs. [19,20]. In order to generalize their work, we consider a more general form for the ac driving, namely

$$
f(t) \equiv \epsilon_{1} \sin \left(\delta t+\delta_{0}\right)+\epsilon_{2} \sin \left(m \delta t+\delta_{0}+\theta\right)
$$

noting that the original formulation is recovered by setting $\delta_{0}=\pi / 2$. Hereafter, we will refer to $\delta_{0}$ as the initial phase and to $\theta$ as the relative phase.

Our collective coordinate theory is based on an Ansatz, proposed in Ref. [29], for the perturbed kink depending on two variables, $X(t)$ and $l(t)$ (position and width of the kink). It is not difficult to show $[22,25,29]$ that the dynamics of these two collective coordinates is given by

$$
\begin{aligned}
& \frac{d P}{d t}=-\beta P-q f(t), \\
& \dot{l}^{2}-2 \ddot{l}-2 \beta l \dot{l}=\Omega_{R}^{2} l^{2}\left[1+\frac{P^{2}}{M_{0}^{2}}\right]-\frac{1}{\alpha},
\end{aligned}
$$

where the momentum $P(t)=M_{0} l_{0} \dot{X} / l(t), \Omega_{R}=1 /\left(\sqrt{\alpha} l_{0}\right)$ with $\alpha=\pi^{2} / 12$ is the so-called Rice frequency, and $M_{0}=8, q=2 \pi$ and $l_{0}=1$ are, respectively, the dimensionless kink mass, topological charge and unperturbed width.

Equation (3) can be solved exactly, and in the large time limit $\left(t \gg \beta^{-1}\right)$ yields

$$
P(t)=-\sqrt{\epsilon}\left[a_{1} \sin \left(\delta t+\delta_{0}-\chi_{1}\right)+a_{2} \sin \left(m \delta t+\delta_{0}+\theta-\chi_{2}\right)\right],
$$

where $\epsilon$ is merely a rescaling parameter in the perturbation expansion, to be determined later; $\chi_{1}=\arctan (\delta / \beta), \quad \chi_{2}=\arctan (m \delta / \beta), a_{1}=q \epsilon_{1} / \sqrt{\epsilon\left(\beta^{2}+\delta^{2}\right)}$, and 
$a_{2}=q \epsilon_{2} / \sqrt{\epsilon\left(\beta^{2}+m^{2} \delta^{2}\right)}$. The change of variable $g(t)^{2}=l(t)$ leads to an Ermakovtype equation for $g(t)$, given by

$$
\ddot{g}+\beta \dot{g}+\left[\left(\frac{\Omega_{R}}{2}\right)^{2}+\left(\frac{\Omega_{R}}{2 M_{0}}\right)^{2} P^{2}(t)\right] g=\frac{1}{4 \alpha g^{3}},
$$

Let us recall that directed motion from ac driving was possible already for a single harmonic in the undamped sine-Gordon system [21], but was suppressed by damping. Therefore, the interesting issue is to find net directed motion from ac driving in the presence of damping, i.e., $\beta \neq 0$. In that case, Eq. (4) cannot be solved $[22,25]$, and therefore we will study it by a perturbative expansion,

$$
l(t)=l_{0}+\epsilon l_{1}(t)+\epsilon^{2} l_{2}(t)+\cdots .
$$

At order $O(\epsilon)$, we obtain from Eq. (6)

$$
\ddot{l}_{1}(t)+\beta \dot{l}_{1}(t)+\Omega_{R}^{2} l_{1}(t)=-\Omega_{R}^{2} P^{2}(t) l_{0} / 2 \epsilon M_{0}^{2} .
$$

If we now substitute the expression of $P(t)$ into (8), we find

$$
\begin{aligned}
\ddot{l}_{1}(t)+\beta \dot{l}_{1}(t)+\Omega_{R}^{2} l_{1}(t) & =A_{1}+A_{2} \cos \left(2 \delta t+2 \delta_{0}-2 \chi_{1}\right) \\
& +A_{3} \cos \left(2 m \delta t+2 \delta_{0}+2 \theta-2 \chi_{2}\right) \\
& +A_{4} \cos \left[(m-1) \delta t+\theta-\left(\chi_{2}-\chi_{1}\right)\right] \\
& -A_{4} \cos \left[(m+1) \delta t+2 \delta_{0}+\theta-\left(\chi_{2}+\chi_{1}\right)\right],
\end{aligned}
$$

where $A_{1}=-A_{2}-A_{3}$, and $A_{2}=\Omega_{R} a_{1}^{2} / 4 \sqrt{\alpha} M_{0}^{2}, A_{3}=\Omega_{R} a_{2}^{2} / 4 \sqrt{\alpha} M_{0}^{2}, A_{4}=$ $-\Omega_{R} a_{1} a_{2} / 2 \sqrt{\alpha} M_{0}^{2}$. By inspection, we conclude from Eq. (9) that $l_{1}(t)$ contains harmonics of frequencies $2 \delta, 2 m \delta$ and $(m \pm 1) \delta$. This is a very important conclusion and we will come back to it below.

After transients elapse, we find

$$
\begin{aligned}
l_{1}(t)= & \frac{A_{1}}{\Omega_{R}^{2}}+\frac{A_{2} \sin \left(2 \delta t+2 \delta_{0}-2 \chi_{1}+\tilde{\theta}_{2}\right)}{\sqrt{\left(\Omega_{R}^{2}-4 \delta^{2}\right)^{2}+4 \beta^{2} \delta^{2}}} \\
& +\frac{A_{3} \sin \left(2 m \delta t+2 \delta_{0}+2 \theta-2 \chi_{2}+\tilde{\theta}_{2 m}\right)}{\sqrt{\left(\Omega_{R}^{2}-4 m^{2} \delta^{2}\right)^{2}+4 m^{2} \beta^{2} \delta^{2}}} \\
& +\frac{A_{4} \sin \left[(m-1) \delta t+\theta-\left(\chi_{2}-\chi_{1}\right)+\tilde{\theta}_{m-1}\right]}{\sqrt{\left(\Omega_{R}^{2}-(m-1)^{2} \delta^{2}\right)^{2}+\beta^{2}(m-1)^{2} \delta^{2}}} \\
& -\frac{A_{4} \sin \left[(m+1) \delta t+2 \delta_{0}+\theta-\left(\chi_{2}+\chi_{1}\right)+\tilde{\theta}_{m+1}\right]}{\sqrt{\left(\Omega_{R}^{2}-(m+1)^{2} \delta^{2}\right)^{2}+\beta^{2}(m+1)^{2} \delta^{2}}},
\end{aligned}
$$

where $\tilde{\theta}_{m}=\arctan \left[\left(\Omega_{R}^{2}-m^{2} \delta^{2}\right) / m \beta \delta\right]$. A cumbersome but otherwise trivial calculation yields the harmonics contained in $l_{2}(t)$, collected in Table 1 .

The next step is to obtain the velocity averaged over one period of the driving. To this end, we use the definition of the momentum, namely

$$
\Pi(t)=\int_{-\infty}^{\infty} d x \phi_{t} \phi_{x}
$$


Table 1. Harmonic content of the first contributions to the perturbative expansion of $l(t)$.

\begin{tabular}{|c|c|c|}
\hline harmonic & $l_{1}$ & $l_{2}$ \\
\hline$m$ & $2 \delta, 2 m \delta,(m \pm 1) \delta$ & $\begin{array}{c}2 \delta, 4 \delta, 2 m \delta, 4 m \delta,(m \pm 1) \delta, \\
2(m \pm 1) \delta,(m \pm 3) \delta,(3 m \pm 1) \delta\end{array}$ \\
\hline 2 & $\delta, 2 \delta, 3 \delta, 4 \delta$ & $\delta, 2 \delta, 3 \delta, 4 \delta, 5 \delta, 6 \delta, 7 \delta, 8 \delta$ \\
\hline 3 & $2 \delta, 4 \delta, 6 \delta$ & $2 \delta, 4 \delta, 6 \delta, 8 \delta, 10 \delta, 12 \delta$ \\
\hline 4 & $2 \delta, 3 \delta, 5 \delta, 8 \delta$ & $\delta, 2 \delta, 3 \delta, 4 \delta, 5 \delta, 7 \delta$, \\
& & $9 \delta, 11 \delta, 13 \delta, 16 \delta$ \\
\hline
\end{tabular}

and find that, for our collective coordinate Ansatz,

$$
\langle\dot{X}(t)\rangle=\frac{1}{T} \int_{0}^{T} \frac{P(t) l(t)}{M_{0} l_{0}} d t .
$$

Considering the expansion (7), this expression can be written as

$$
\begin{aligned}
\langle\dot{X}(t)\rangle & =\frac{1}{T} \int_{0}^{T} \frac{P(t)\left(l_{0}+\epsilon l_{1}(t)+\epsilon^{2} l_{2}(t)+\ldots\right)}{M_{0} l_{0}} d t \\
& =\left\langle\dot{X}_{0}(t)\right\rangle+\epsilon\left\langle\dot{X}_{1}(t)\right\rangle+\epsilon^{2}\left\langle\dot{X}_{2}(t)\right\rangle+\ldots
\end{aligned}
$$

At $O\left(\epsilon^{0}\right)$, the averages $\langle P(t)\rangle$ and $\left\langle\dot{X}_{0}(t)\right\rangle$ vanish trivially; therefore, net kink motion can only arise in next order. By straightforward calculations from Eqs. (10) and (12) we find for $m=2$ that, for large enough times,

$$
\begin{aligned}
\epsilon\left\langle\dot{X}_{1}\right\rangle=\frac{q^{3} \Omega_{R}^{2} \epsilon_{1}^{2} \epsilon_{2}}{8 M_{0}^{3}\left(\beta^{2}+\delta^{2}\right) \sqrt{\beta^{2}+4 \delta^{2}}} & \left(\frac{2 \cos \left[\delta_{0}-\theta+\left(\chi_{2}-2 \chi_{1}\right)-\tilde{\theta}_{1}\right]}{\sqrt{\left(\Omega_{R}^{2}-\delta^{2}\right)^{2}+\beta^{2} \delta^{2}}}\right. \\
& \left.-\frac{\cos \left[\delta_{0}-\theta+\left(\chi_{2}-2 \chi_{1}\right)+\tilde{\theta}_{2}\right]}{\sqrt{\left(\Omega_{R}^{2}-4 \delta^{2}\right)^{2}+4 \beta^{2} \delta^{2}}}\right) .
\end{aligned}
$$

It is now clear that, for Eq. (14) to be valid, and our perturbative expansion (7) to hold, as $\epsilon$ has to be small, the prefactor on the right-hand side of (14) has to be much smaller than 1, which establishes a priori the range of applicability of our theory (although generally speaking collective coordinate theories usually apply for larger perturbations than predicted [10]). This is the final result of the collective coordinate approach. We now proceed to discuss the predictions we can deduce from this expressions and to verify the results by comparing to numerical simulations.

\section{Discussion}

To begin with, the result obtained in Eq. (14) predicts that for $m=2$ kinks should travel with a non-zero velocity, in agreement with the reports in Refs. $[19,20]$. If we fix $\delta_{0}=\pi / 2$, the case studied by those authors, we find a sinusoidal dependence on the relative phase $\theta$ exactly as reported in those previous works. At some specific values of this relative phase, the velocity vanishes, meaning that parameters can be 


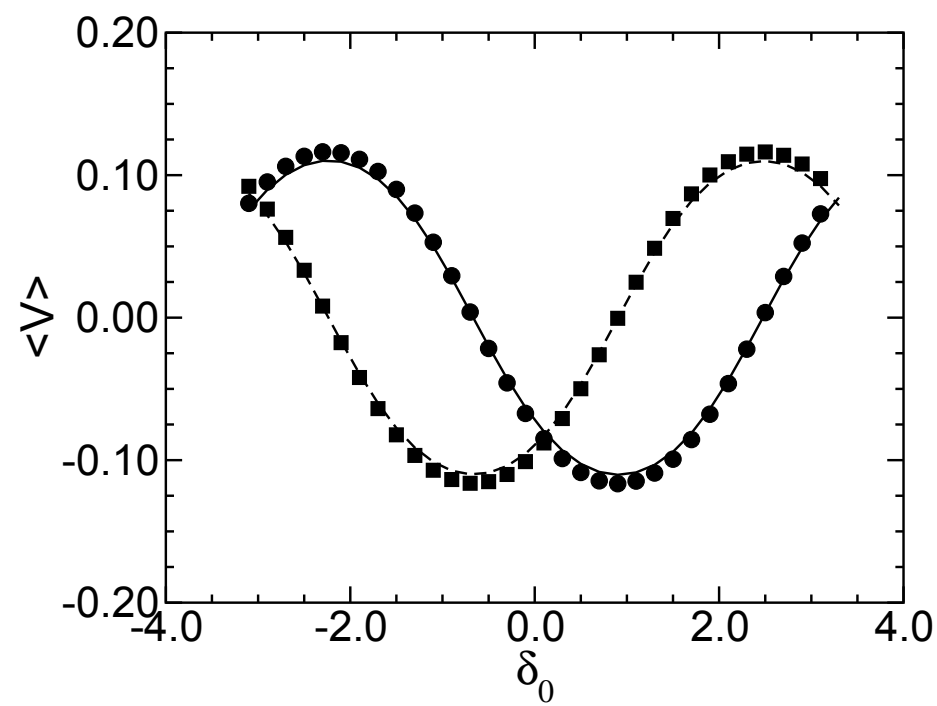

Fig 1. Dependence of the kink velocity on the initial phase. Parameters are $\epsilon_{1}=\epsilon_{2}=0.2$, $\beta=0.05, \delta=0.1$. Relative phase $\theta=\pi / 2$ : solid line, CC theory; filled circles, simulation results. Relative phase $\theta=0$ : dashed line, $\mathrm{CC}$ theory; squares, simulation results.

chosen as to move in any desired direction or to keep the kink oscillating around some point.

Secondly, the expression (14) has more implications. Indeed, it can be seen that the velocity has an additional sinusoidal dependence on the initial phase, $\delta_{0}$. This is a new result, not discussed by Flach et al. and Salerno and Zolotaryuk, that shows that relative and initial phase play exchangeable roles. That can be immediately seen by a change of variables in the original sine-Gordon equation (1): taking $\delta_{0}$ to be $\delta t_{0}$, we find that changing the time variable in the fashion $t^{\prime}=t+t_{0}$ leads to the conclusion that an initial phase $\delta_{0}$ is equivalent to a relative phase $\theta^{\prime}=\theta-(m-1) \delta_{0}$ for a kink displaced from $x_{0}$ to $x_{0}+V t_{0}$.

Before proceeding any further, it is important to check the validity of the two conclusions we have just presented. After all, our collective coordinate theory is basically a variational approach, and in addition we have also treated perturbatively the resulting equations. Therefore, numerical simulations of the full sine-Gordon equation (1) are needed to verify the predictions of the calculations in the previous section. To this end, we carried out such simulations (see Ref. [28] for details on the numerical procedure) and the outcome is plotted in Fig. 1. We see that the agreement between theory and simulation is perfect, and there indeed is the double dependence on both the initial and the relative phase as found in Eq. (14). This fully confirms our approach and even our predictions in a quantitative manner.

At this point, it is interesting to consider the following issue, which arises if we want to consider our model as a ratchet: Looking at the sinusoidal dependence on the initial phase, it may be concluded that the system is not a ratchet because an average over any possible initial phase would in principle lead to zero mean velocity. 
However, this is not the case: Recall that, as mentioned in the previous paragraph, changing the initial phase implies changing the relative phase as well. Then we can see, by inspecting Eq. (14), that the kink velocity depends on the difference $\delta_{0}-\theta$, which is the same for all choices of $\delta_{0}$. Hence, the kink velocity is the same for all $\delta_{0}$, and averaging does not suppress the ratchet effect.

With the $m=2$ results of our approach well established by the comparison with the simulations, let us look at the $m=3$ case. Once again, our theoretical results agree with and explain the numerical findings in Refs. $[19,20]$ : the prediction from Eq. (14) is that the velocity is zero and therefore that there is no net directed motion of the kink, as observed in those previous papers. To be sure, we have observed that if the driving force is strong enough (not for any driving value, as in the ratchet effect) directed motion is observed (as has been reported in [20]), but the system is so largely distorted that one cannot conclude that the kink is moving as a coherent entity (it looks as though the motion is driven by the kink wings), and therefore that is an altogether different question.

Why is net directed motion suppressed for $m=3$ ? The explanation of this rule is transparent from Table 1 and constitutes the most relevant success of our approach. For $m=3$, the frequencies of the ac force (or the momentum) are odd harmonics of $\delta$, whereas the width of the kink (governed by the internal mode) oscillates only with even harmonics. Figure 2 shows clearly and with very high accuracy that the simulations behave exactly as predicted by the collective coordinate equations. Note that in Fig. 2 we plot results obtained for $m=4$ as well, a situation in which we recover the ratchet effect and the kink moves towards a preferred direction for any value of the ac driving.

This remark directs us to both the mechanism responsible for the appearance of directed motion and the statement of a selection rule based on it. The mechanism is the coupling between the translation of the kink and the internal mode. This is reflected in Eqs. (3) and (4), which show that the ac driving $f(t)$ acts on the kink width through $P^{2}(t)$, whereas $P(t)$ itself is in turn inversely proportional to $l(t)$. This coupling is the responsible for the net kink motion, but for it to be actually possible, the harmonic content of the effective force $P^{2}(t)$ acting on the width degree of freedom must be able to resonate with it. This is evident from Eq. (12), in which the integral is nonzero only if $l(t)$ contains at least one of the harmonics of $P(t)$. It is important to realize that this condition is much more restrictive than that found in Ref. [24], where only the necessity of $l(t)$ being a dynamic variable was pointed out, but no conditions on its frequency content or any other feature were obtained. We have just seen that this is indeed necessary, but that additional, crucial resonance conditions have to be fulfilled.

\section{Effects of noise}

As a final step to set our results on firm grounds, we studied the robustness of the kink directed motion against noisy perturbations (much in the same way as in Ref. [20]). To this end, we considered the Langevin equation

$$
\phi_{t t}-\phi_{x x}+\sin (\phi)=-\beta \phi_{t}+f(t)+\xi(x, t)
$$



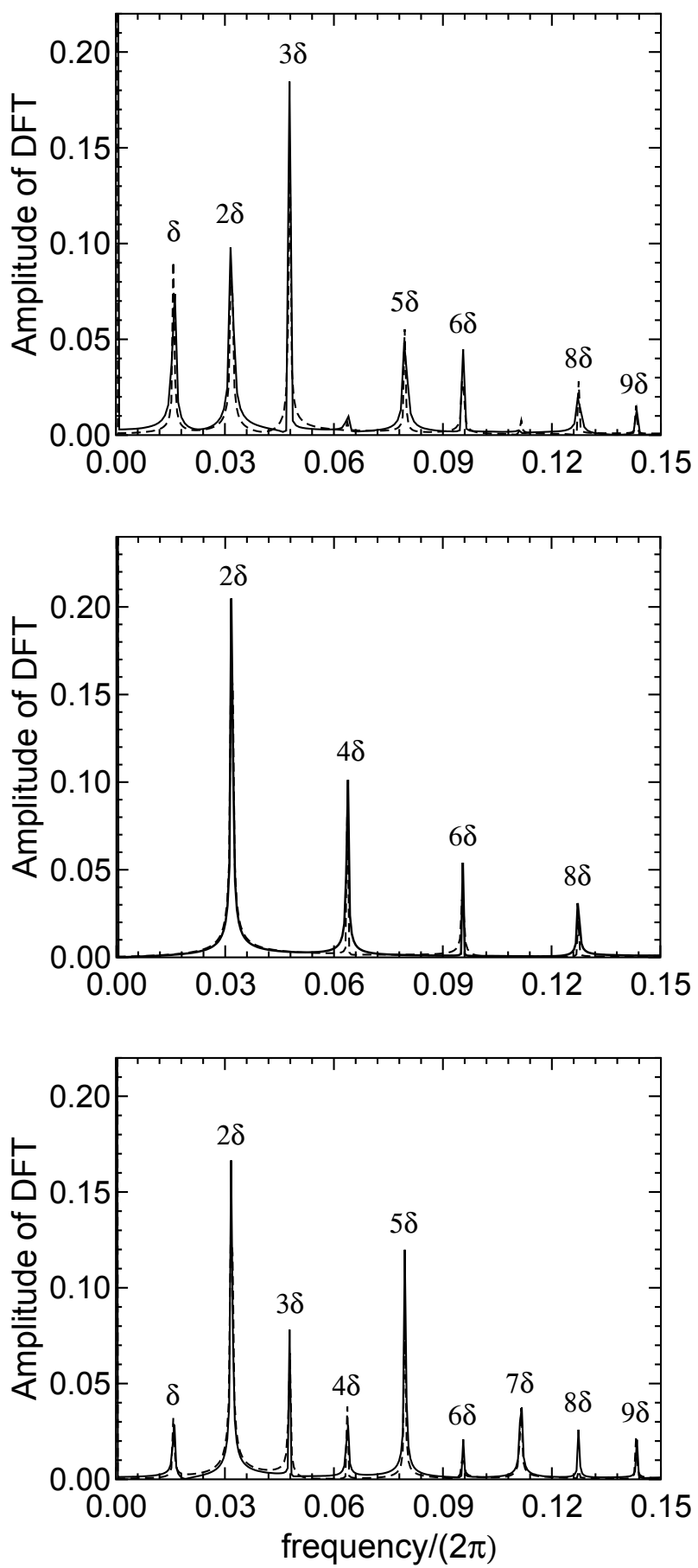

Fig 2. Discrete Fourier Transform of the kink width. Upper panel: $m=2$; middle panel: $m=3$; lower panel: $m=4$. Solid line: amplitude measured in simulations. Dashed line: numerical integration of the $\mathrm{CC}$ equations. Parameters are as in Fig. 1 for relative phase $\theta=\pi / 2$ and $\delta_{0}=-2.5$. 


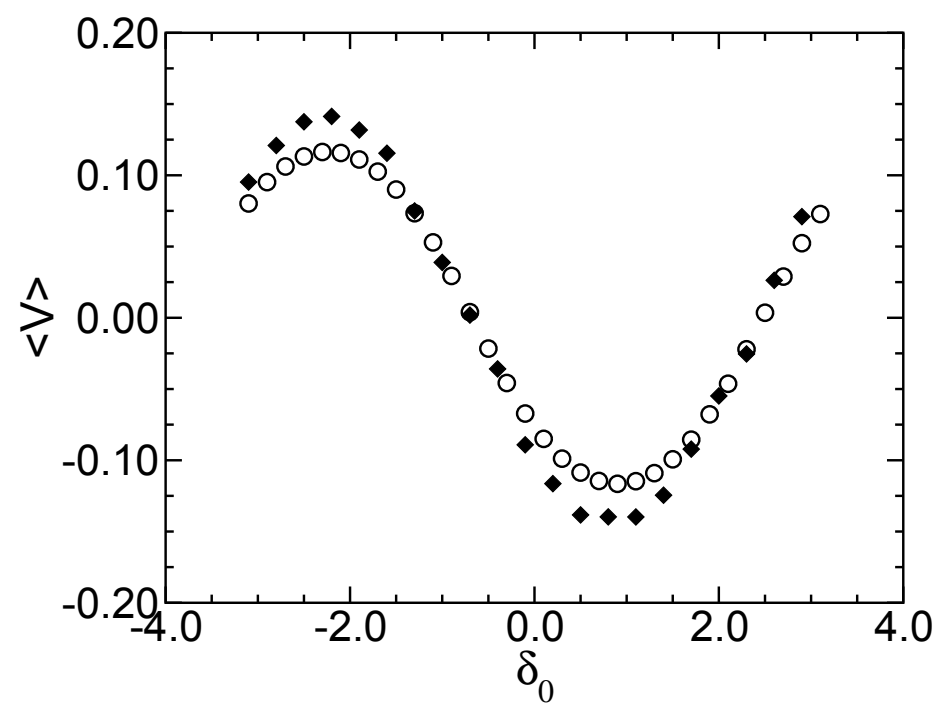

Fig 3. Dependence of the kink velocity on the initial phase for relative phase $\theta=\pi / 2$ in the deterministic $(D=0$, empty circles) and the stochastic $(D=0.03$, diamonds $)$ cases. Other parameters are as in Fig. 1.

where $\xi(x, t)$ is a gaussian white noise of zero mean and correlations $\left\langle\xi(x, t) \xi\left(x^{\prime}, t^{\prime}\right)\right\rangle=$ $D \delta\left(x-x^{\prime}\right) \delta\left(t-t^{\prime}\right)$. In this case, the equation was studied only by numerical simulations, which were carried out using a Heun scheme [30]. While one could in principle think that this noise would suppress the initial phase dependence, Fig. 3 shows that the opposite is the case: the noise enhances the dependence on the initial phase, increasing the maximum values of the velocity while keeping the same general sinusoidal dependence and the location of the zeros. It is tempting to conclude from this plot that the noise, at least if it is not very large, assists the process of energy transfer between the width and the translation degrees of freedom, activating it.

Having verified, as intended, that the ratchet effect is robust to the effects of noise, we find it interesting to look any further on this activation phenomenon in its own right. Therefore, we looked at one particular point in Fig. (3) where the velocity was larger (in absolute value) and performed numerical simulations varying only the noise intensity, leaving all other parameters unchanged. The results are summarized in Fig. 4. The first conclusion we can draw from this plot is that the noise has indeed a nontrivial activation effect on the kink motion. As can be seen, the velocity exhibits an increasing trend with the noise intensity, although the dependence is rather noisy. To verify the trend we have made two linear fits to the two sets of simulation data, finding comparable slope values of 0.053 and 0.060 . Upon increasing the noise further, we found that kink-antikink pairs were spontaneously generated in the system, which eventually collide with the original one and render the simulation useless (from the viewpoint of measuring the kink velocity). However, it is clear that if we were able to study even larger values of the noise, the velocity has to decrease as diffusion becomes the dominant process, effectively sup- 


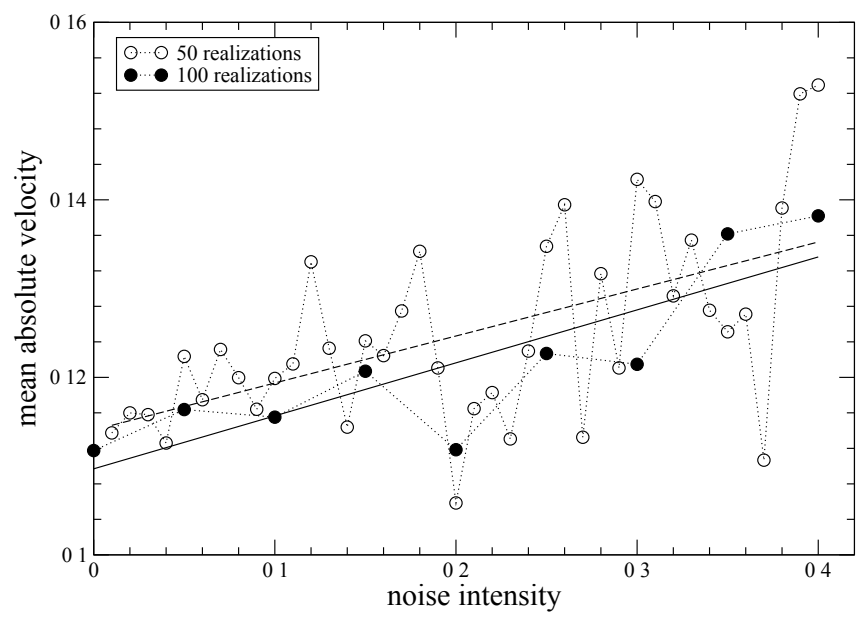

Fig 4. Mean absolute value of the kink center velocity vs noise intensity. Parameters are $\epsilon_{1}=\epsilon_{2}=$ $0.2, \beta=0.05, \delta=0.1$, initial phase $\delta_{0}=-0.31831$, and relative phase $\theta=-\pi / 2$. Realizations in the averages are as indicated. The straight lines are linear fits of slopes 0.053 (dashed, for the 50 realizations set) and 0.060 (solid, for the 100 realizations set).

pressing the ac driving effects. In that case, asymptotically, the velocity must go to zero as noise increases, and we would be observing a manifestation of a phenomenon of much interest during the last decade, namely stochastic resonance. Stochastic resonance was (very much like ratchets) first found in point-like systems [31], but it has been generalized to extended systems in the last few years (see, e.g., Ref. [32]). In this respect, it is interesting to recall Ref. [33], where thermal resonances as a function of temperature were observed, to our knowledge for the first time. By comparing the system in that previous work with the present one, we believe that we can indeed expect the appearance of resonant phenomena. We thus plan to carry out further simulations with different parameters, in particular, with larger damping, in order to suppress the nucleation of kink-antikink pairs and be able to observe the dynamics of a single kink for larger noises. Analytical calculations will also be desirable to ascertain the nature of this activation process. In any event, what we have established is that net directed motion can be observed even for moderate and large noise intensities, which shows that the mechanism proposed here is extraordinarily robust. Work along these lines is in progress.

\section{Conclusions}

Regarding the deterministic part, the main conclusion of this work is the understanding of the condition for the appearance of net directed motion in an ac driven, damped sine-Gordon system. Our collective coordinate theory, thoroughly confirmed by the numerical simulations of the full partial differential equation, has 
allowed us to show that the motion arises from the coupling of the internal mode (effectively arising from odd phonon contributions) to the translation of the kink. As a byproduct, we have found the physical reasons for the symmetry requirements first proposed in Refs. [19,20] for the existence of this ratchet-like phenomenon. Indeed, to make net motion possible, the indirect forcing arising from the internal mode influence has to resonate with the available frequencies for the width of the kink itself. Interestingly, while this phenomenon should be expected in any other soliton-bearing model with solitons possessing an internal mode (e.g., the $\phi^{4}$ model), for the sine-Gordon equation it is an apparent puzzle due to its lack of internal modes [25]. However, as explained above, (odd) phonons have been shown to give rise to width oscillations very similar to those induced by an internal mode [26]. We believe that what we are seeing is precisely the result of the action of those phonons, summarized in our approach through the width variable $l(t)$. This conclusion reinforces, in turn, the interpretation of Quintero and Kevrekidis [26], which will probably be useful in other instances of sine-Gordon problems where kink oscillations play a role. Our analytical calculations have also clarified the influence of the relative phase and the initial phase, establishing their equivalence. In this respect, we should stress the fact that the dependence of the kink velocity on the initial phase does not suppress the ratchet effect, due precisely to this relationship with the relative phase and the result that the velocity is unchanged when changing the initial phase.

With respect to the effects of noise, the conclusions are not that definite. We have certainly established the robustness of the ratchet effect under the influence of noise, even for rather large noise values. In addition, we have verified that for moderate noises, the velocity of the directed motion increases with noise intensity, up to the point in which thermally generated kink-antikink pairs do not allow us to follow a single kink. The existence of this activation process along with the fact that diffusion must dominated at larger noises have led us to conjecture that the system could exhibit some form of stochastic resonance. In order to confirm this interpretation, much more numerical and analytical work is needed. Apparently, the motion takes place through the same mechanism, so the two collective coordinate equations are likely to provide a good starting point to understand the phenomenon. However, it is also known that the diffusion of sine-Gordon solitons is controlled by a diffusion constant which depends both linearly and quadratically on the noise intensity in the full partial differential equation [34]. This is another factor that could be playing a role in the observed nonmonotonous dependence of the velocity on the noise. It is then clear that the problem is still far from understood and that we cannot offer a final conclusion about it at this stage.

From a more applied point of view, this work may have important consequences for applications as a way of separating, e.g., fluxons in long Josephson junctions [15]. Interestingly, such superconducting devices (and related ones, see e.g. Ref. [3] and references therein) provide the best possible laboratory to verify our results. This experimental confirmation is crucial in order to ascertain their applicability. Given the accuracy with which the sG equation describes long Josephson junctions, and the fact that an external force like the one proposed in this and earlier works $[19,20]$ is easy to implement, we hope that the corresponding measurements will soon be carried out. As a bonus, the possible existence of stochastic resonance 
would then be easier to explore experimentally. Finally, the applicability of this internal mode mechanism may have other, far-reaching implications in very different kinds of systems. Indeed, the original motivation of this work is the modelling of molecular motors such as kinesin. It is well known now that kinesin "walks" along microtubules by simultaneously changing its shape (see Ref. [35] and references therein) to allow one "head" to overcome the other. The internal mode mechanism proposed here is clearly reminiscent of the (obviously much more complicated) way kinesin moves. As another example, we want to mention the recent experiments on kink-induced transport in granular media by Moon et al. [36], in which it has been observed directed motion of kinks separating convection rolls when the system is under the influence of two harmonic drivings similar to those considered here. While the direct comparison of our simple model with these phenomena is out of the question, we do believe that the paradigm of coupling to internal modes could help understand the experiments from a more mesoscopic viewpoint.

\section{Acknowledgments}

AS has been supported by the Ministerio de Ciencia y Tecnología of Spain through grant BFM2003-07749-C05-01. NRQ has been supported by the Ministerio de Ciencia y Tecnología of Spain through grant BFM2001-3878-C02, by the Junta de Andalucía under project FQM-0207, by DAAD (Germany) A0231253/Ref. 314, and, along with LMM and FGM, by the International Research Training Group 'Nonequilibrium Phenomena and Phase Transitions in Complex Systems' (DFG, Germany). KL has been supported by the Engineering Research Program of the Office of Basic Energy Sciences at the U. S. Department of Energy under Grant No. DE-FG0386ER13606.

\section{References}

[1] A. C. Scott, Nonlinear Science (Oxford University, Oxford, 1999).

[2] P. Reimann, Phys. Rep. 361, 57 (2002).

[3] R. Dean Astumian and P. Hänggi, Phys. Today 55 (11), 33 (2002).

[4] F. Jülicher, A. Adjari and J. Prost, Rev. Mod. Phys. 69, 1269 (1997).

[5] H. Linke, ed., Ratchets and Brownian Motors: Basics, Experiments and Applications, Appl. Phys. A 75 (2002), special issue.

[6] R. P. Feynman, R. B. Leighton, M. Sands, The Feynman Lectures in Physics, Vol. I, ch. 46 (Addison-Wesley, Reading, 1963).

[7] M. O. Magnasco, Phys. Rev. Lett. 71, 1477 (1993).

[8] J. Prost, J.-F. Chauwin, L. Peliti and A. Adjari, Phys. Rev. Lett. 72, 2652 (1994).

[9] P. Jung, J. G. Kissner, and P. Hänggi, Phys. Rev. Lett. 76, 3436 (1996); T. E. Dialynas, K. Lindenberg, and G. P. Tsironis, Phys. Rev. E 56, 3976 (1997); J. L. Mateos, Phys. Rev. Lett. 84, 258 (2000).

[10] A. Sánchez and A. R. Bishop, SIAM Rev. 40, 579 (1998).

[11] F. Marchesoni, Phys. Rev. Lett. 77, 2364 (1996).

[12] A. V. Savin, G. P. Tsironis and A. V. Zolotaryuk, Phys. Lett. A 229, 279 (1997); Phys. Rev. E 56, 2457 (1997).

[13] P. Reimann, R. Kawai, C. Van den Broeck, and P. Hänggi, Europhys. Lett. 45, 545 (1999); C. Van den Broeck, I. Bena, P. Reimann, and J. Lehmann, Ann. Phys. 
(Leipzig) 9, 713 (2000); J. Buceta, J. M. Parrondo, C. Van den Broeck, and F. J. de la Rubia, Phys. Rev. E 61, 6287 (2000); G. Costantini and F. Marchesoni, Phys. Rev. Lett. 87, 114102 (2001); Z. Zheng, M. C. Cross, and G. Hu, Phys. Rev. Lett. 89, 154102 (2002); G. Costantini, F. Marchesoni, and M. Borromeo, Phys. Rev. E 65, 051103 (2002); L. M. Floría, F. Falo, P. J. Martínez, and J. J. Mazo, Europhys. Lett. 60, 174 (2002).

[14] M. Salerno and N. R. Quintero, Phys. Rev. E 65, 025602 (2002).

[15] G. Carapella and G. Costabile, Phys. Rev. Lett. 87, 077002 (2001); E. Goldobin, A. Sterck, and D. Koelle, Phys. Rev. E 63, 031111 (2001); F. Falo, P. J. Martínez, J. J. Mazo, T. P. Orlando, K. Segall, E. Trías, in Ref. [5], p. 263.

[16] S. Flach, O. Yevtushenko, and Y. Zolotaryuk, Phys. Rev. Lett. 84, 2358 (2000).

[17] I. Goychuk and P. Hänggi, in Stochastic Processes in Physics, Chemistry and Biology, edited by J. A. Freund and T. Pöschel (Lecture Notes in Physics, no. 557, SpringerVerlag, Berlin, 2000).

[18] I. Goychuk and P. Hänggi, J. Phys. Chem. B 105, 6642 (2001).

[19] S. Flach, Y. Zolotaryuk, A. E. Misoshnichenko, and M. V. Fistul, Phys. Rev. Lett. 88, 184101 (2002).

[20] M. Salerno and Y. Zolotaryuk, Phys. Rev. E 65, 056603 (2002).

[21] N. R. Quintero and A. Sánchez, Phys. Lett. A 247, 161 (1998); Eur. Phys. J. B 6, 133 (1998).

[22] N. R. Quintero, A. Sánchez, and F. G. Mertens, Phys. Rev. Lett. 84, 871 (2000).

[23] N. R. Quintero, A. Sánchez, and F. G. Mertens, Phys. Rev. E 62, 5695 (2000).

[24] C. R. Willis and M. Farzaneh, arXiv: cond-mat/0212125.

[25] N. R. Quintero, A. Sánchez, and F. G. Mertens, Phys. Rev. E 62, R60 (2000).

[26] N. R. Quintero and P. G. Kevrekidis, Phys. Rev. E 64, 056608 (2001).

[27] S. Cilla, F. Falo, and L. M. Floría, Phys. Rev. E 63, 031110 (2001).

[28] L. Morales-Molina, N. R. Quintero, F. G. Mertens, and A. Sánchez, Phys. Rev. Lett. 91, 234102 (2003).

[29] M. Salerno and A. C. Scott, Phys. Rev. B 26, 2474 (1982); M. J. Rice, Phys. Rev. B 28, 3587 (1983).

[30] M. San Miguel and R. Toral, in Nonequilibrium Structures VI, edited by E. Tirapegui (Kluwer, Dordrecht, 1998).

[31] L. Gammaitoni, P. Hänggi, P. Jung, and F. Marchesoni, Rev. Mod. Phys. 70, 223 (1998).

[32] J. García-Ojalvo and J. M. Sancho, Noise in spatially extended systems (SpringerVerlag, New York, 1999).

[33] R. Reigada, A. Sarmiento, and K. Lindenberg, Phys. Rev. E 63, 66113 (2001).

[34] N. R. Quintero, A. Sánchez and F. G. Mertens Eur. Phys. J. B 16, 361 (2000).

[35] A. Yildiz, M. Tomishige, R. D. Vale and P. R. Selvin, Science 303, 676 (2004).

[36] S. J. Moon, D. I. Goldman, J. B. Swift, and H. L. Swinney, Phys. Rev. Lett. 91, 134301 (2003). 